\title{
A NOTE ON ZERO DIVISORS IN GROUP-RINGS
}

\author{
JACQUES LEWIN ${ }^{1}$
}

\begin{abstract}
Let $Z G_{1}$ and $Z G_{2}$ be the integral group rings of groups $G_{1}$ and $G_{2}$ with a common normal subgroup $H$ and let $K$ be a subgroup of $H$. Let $G$ be the free product of $G_{1}$ and $G_{2}$ amalgamating $K$. If $Z G_{1}$ and $Z G_{2}$ are integral domains and if $Z H$ has the Ore condition then $Z G$ is again an integral domain.
\end{abstract}

In this paper we apply a theorem of P. M. Cohn [1] to show that the group-ring of some generalized free products of groups has no zero divisors.

Recall that a ring $R$ without zero divisors has the right Ore condition if any two nonzero elements of $R$ have a common nonzero right multiple. In this situation $R$ has a uniquely determined skew field $D$ of right quotients: every element of $D$ is of the form $x y^{-1}$ with $x, y \in R$. (See e.g. [2, Theorem 1.3].)

Let us agree, by abus de language, to say that the group $G$ has no zero divisors if the group-ring $Z G$ has no zero divisors. Let $H$ be a subgroup of the group $G$ without zero divisors, and suppose $Z G$ has the right Ore condition. (We note in passing that the right and left Ore condition are equivalent for group-rings.) Then $Z H$ also has the right Ore condition. For $Z G$ is a free right $Z H$ module freely generated by a right transversal of $G$ modulo $H$. Thus if $x, y$ are in $Z H$ there are nonzero elements $t, u$ in $Z G$ with $x t=y u$. We need only consider this equation coset per coset to find nonzero elements $t^{\prime}$ and $u^{\prime}$ in $Z H$ with $x t^{\prime}=y u^{\prime}$. This said, we may proceed to our results.

THEOREM 1. Let $G_{i}(i=1,2)$ be a group without zero divisors, and let $H_{i}$ be a normal subgroup of $G_{i}$ such that $Z H_{i}$ has the right Ore condition. Let $K$ be a common subgroup of $H_{1}$ and $H_{2}$ and let $G$ be the generalized free product of $G_{1}$ and $G_{2}$ amalgamating $K$. Then $Z G$ has no zero divisors.

Proof. Let $D_{i}$ be the fields of quotients of $Z H_{i}$, and consider the abelian group $R_{i}=Z G_{i} \otimes_{z H_{i}} D_{i}$. We turn $R_{i}$ into a ring by defining

$$
\left(g_{1} \otimes l_{1}^{-1}\right)\left(g_{2} \otimes l_{2}^{-1}\right)=g_{1} g_{2} \otimes\left(g_{2}^{-1} l_{1} g_{2}\right)^{-1} l_{2}^{-1}
$$

Received by the editors January 15, 1971.

AMS 1970 subject classifications. Primary 16A26; Secondary 20E30.

Key words and phrases. Group-rings, zero divisors, free products.

1 This work was supported by NSF Grant GP-8094.

(C) American Mathematical Society 1972 
for $g_{1}, g_{2} \in G_{i}, l_{1}, l_{2} \in Z H_{i}$, and extending by linearity. We leave it to the reader to verify that this is well defined. (Let $s \in Z H, r \in Z G, r=\sum \alpha_{i} g_{i}$. Then there are elements $x_{i} \in Z H$, such that $g_{i}^{-1} s g_{i} x_{i}=t$, for some $t \in Z H$. Thus $s \sum \alpha_{i} g_{i} x_{i}=\sum \alpha_{i} g_{i} g_{i}^{-1} s g_{i} x_{i}=\sum \alpha_{i} g_{i} t=r t$. Thus $Z H-\{0\}$ is a right divisor set in $Z G$ (see again [2]) and by Ore's theorem $Z G$ has a ring of right quotients with respect to $Z H$. Our definition gives a concrete representation for this ring of quotients.)

Since $Z G_{i}$ is a free $Z H_{i}$ module, the map $Z G_{i} \rightarrow Z G_{i} \otimes 1$ is a monomorphism. Further $R_{i}$ has no zero divisors. For $\left(x_{1} \otimes d_{1}^{-1}\right)\left(x_{2} \otimes d_{2}^{-1}\right)=0$ only if $\left(1 \otimes d_{1}^{-1}\right)\left(x_{2} \otimes 1\right)=0$ which forces $x_{2} \otimes 1=0$ since $1 \otimes d_{1}^{-1}$ is invertible.

Now, as we pointed out, $Z K$ has the Ore condition and its skew field $D$ of quotients is contained in $D_{i}$. We now consider $R_{i}$ as a right $D$ vector space by identifying $D$ with $1 \otimes D$. Let $\mathscr{S}_{i} \cup\{1\}$ be a right tranversal for $K$ in $G_{i}$ and suppose that $\sum_{j}\left(s_{j} \otimes 1\right) d_{j}=0$ with the $s_{j}$ distinct elements of $\mathscr{S}_{1} \cup\{1\}$, say, and $d_{i} \in D$. Then, for some $d_{j}^{\prime}$ and $d$ in $Z K, d_{j}=d_{j}^{\prime} d^{-1}$, and thus $\sum_{j}\left(s_{j} \otimes 1\right) d_{j}^{\prime}=\sum_{j}\left(s_{j} d_{j}^{\prime} \otimes 1\right)=0$. It follows that the $d_{j}^{\prime}$, and hence the $d_{j}$, are all zero. Thus $\mathscr{S}_{i} \cup\{1\}$ may be extended to a basis $B_{i} \cup\{1\}$ of $R_{i}$ qua $D$ vector space.

We now form the free product $R$ (qua rings) of $R_{1}$ and $R_{2}$ amalgamating $D$. By Cohn's theorem, $R$ has no zero divisors and the set $B$ of monomials on the alphabet $B_{1} \cup B_{2}$ (with consecutive letters in different factors) forms, together with 1 , a $D$ basis for $R$.

It remains to show that $Z G$ is contained in $R$. To this effect we need only show that the normal forms $s_{i_{1}} s_{i_{2}} \cdots s_{i_{k}} k$ with $k \in K$ and no two $s_{i_{j}}$, $s_{i_{j+1}}$ in the same $\mathscr{S}_{i}$ and $\boldsymbol{Z}$ independent. This is however an immediate consequence of the $D$ independence of $B$.

Group-rings with the Ore condition are fairly common, as the following shows:

Proposition. Let $G$ be a solvable group without zero divisors. Then $Z G$ has the (right) Ore condition.

Proof. It is clearly sufficient to prove the proposition for finitely generated $G$. Then there is a finite normal series with cyclic factors between $[G, G]$ and $G$. Using induction both on the length of this series and on the solvability length of $G$ we may assume that $G$ has a normal subgroup $H$ with $G / H$ cyclic and such that $Z H$ has the Ore condition.

Let $a$ generate $G$ modulo $H$ and let $x$ and $y$ be nonzero elements of $Z G$, say $x=\sum_{i=0}^{n} h_{i} a^{i}, y=\sum_{i=0}^{m} k_{i} a^{i}$ with $h_{i}, k_{i} \in Z H$ and $n \geqq m$. (Since $a$ is a unit in $Z G$, it is clear that we need only consider elements involving positive powers of $a$.) By assumption there exist $k_{n}^{\prime}$ and $h_{m}^{\prime}$ with $h_{n} k_{n}^{\prime}=k_{m} h_{m}^{\prime}$. Let 
$M$ be the matrix

$$
M=\left(\begin{array}{cc}
1 & 0 \\
a^{-n} k_{n}^{\prime} a^{n} & -a^{-m} h_{m}^{\prime} a^{m} a^{n-m}
\end{array}\right) .
$$

Then, if $\left(\begin{array}{l}x^{\prime} \\ y^{\prime}\end{array}\right)=M\left(\begin{array}{l}x \\ y\end{array}\right)$ we may assume by induction on $n+m$ that there is a row vector $\left(t_{1}, t_{2}\right)$ with nonzero entries such that $\left(t_{1}, t_{2}\right)\left(\begin{array}{l}x^{\prime} \\ y^{\prime}\end{array}\right)=0$. If we set $\left(t_{1}^{\prime}, t_{2}^{\prime}\right)=\left(t_{1}, t_{2}\right) M$, then $\left(t_{1}^{\prime}, t_{2}^{\prime}\right)$ is a nonzero vector with $\left(t_{1}^{\prime}, t_{2}^{\prime}\right)\left(\begin{array}{l}x \\ y\end{array}\right)=0$, as required.

CONJECTURE. If $Z G$ has no zero divisors and does not have the Ore condition, then $G$ contains a free (noncyclic) subgroup.

THEOREM 2. Let $G_{i}(i=1,2)$ be a group such that $Z G_{i}$ is embeddable in a skew field $D_{i}$ and $H$ be a subgroup of $G_{i}$ such that $Z H$ has the Ore condition. If $G$ is the free product of $G_{1}$ and $G_{2}$ amalgamating $H$, then $Z G$ has no zero divisors.

Proof. Both $D_{1}$ and $D_{2}$ contain the quotient skew field $D$ of $Z H$ and we may form the free product $R$ of $D_{1}$ and $D_{2}$ amalgamating $D$. Proceeding as in the proof of Theorem 1, we find that $Z G$ is contained in $R$ which has no zero divisors.

The above results extend an unpublished theorem of G. Baumslag (who proved that the free product of two residually torsion free nilpotent groups amalgamating a cycle has no zero divisors) and overlap with some work of A. Karrass and D. Solitar (also unpublished) who proved that the free product of two locally indicable groups amalgamating a cycle is again locally indicable.

\section{REFERENCES}

1. P. M. Cohn, On the free product of associative rings. III, J. Algebra 8 (1968), 376-383. MR 36 \#5170.

2. A. V. Jategaonkar, Left principal ideal rings, Lecture Notes in Math., no. 123, Springer-Verlag, Berlin and New York, 1970.

Department of Mathematics, Syracuse University, Syracuse, New York 13210 